\title{
Photocurrent readout and electro-optical tuning of resonantly excited exciton polaritons in a trap
}

\author{
K. Winkler, ${ }^{1}$ P. Gold, ${ }^{1}$ B. Bradel, ${ }^{1}$ S. Reitzenstein, ${ }^{2}$ V. D. Kulakovskii, ${ }^{3}$ M. Kamp, ${ }^{1}$ C. Schneider, ${ }^{1}$ and S. Höfling ${ }^{1,4}$ \\ ${ }^{1}$ Technische Physik, Physikalisches Institut and Wilhelm Conrad Röntgen-Research Center for Complex \\ Material Systems, Universität Würzburg, Am Hubland, D-97074, Würzburg, Germany \\ ${ }^{2}$ Institut für Festkörperphysik, Technische Universität Berlin, Hardenbergstrasse 36, 10623 Berlin, Germany \\ ${ }^{3}$ Institute of Solid State Physics, Russian Academy of Science, Chernogolovka 142432, Russia \\ ${ }^{4}$ SUPA, School of Physics and Astronomy, University of St. Andrews, St. Andrews KY16 9SS, United Kingdom
}

(Received 25 July 2014; revised manuscript received 8 October 2014; published 20 January 2015)

\begin{abstract}
We discuss a technique to manipulate and read out strictly resonantly excited exciton polaritons confined in a three-dimensional trap. The polaritons are trapped via their photonic part in a locally elongated microcavity with a high quality factor $(Q \sim 6000)$, giving rise to sharp zero-dimensional resonances. Manipulation of the polaritons is achieved by spectrally tuning the quantum well excitons via the quantum confined Stark effect up to $10 \mathrm{meV}$, while the signal of the resonantly excited polaritons is simultaneously read out via the photocurrent flowing through the device. The effects of polariton-polariton interactions and interactions with the environment are revealed in the zero detuning regime by excitation power dependent investigations. By increasing the polariton number in our trap via resonant optical injection, we observe a pronounced blueshift of the lower polariton eigenenergy towards the weakly coupled cavity resonance. Furthermore, the photocurrent exhibits pronounced nonlinearities when self-tuned into resonance via its excitation dependent spectral blueshift.
\end{abstract}

DOI: 10.1103/PhysRevB.91.045127

PACS number(s): 78.67.De, 71.35.Lk, 73.50.Pz

Exciton polaritons are fascinating bosonic quasiparticles, arising from the strong coupling between quantum well (QW) excitons and photons confined in a semiconductor microcavity. They are extremely appealing for studying the fundamentals of bosonic physics, such as Bose-Einstein condensation related effects, even at very high temperatures (up to $300 \mathrm{~K}$ ) [1-8]. Moreover, polaritons have also been proposed to be promising candidates for the generation of single and indistinguishable photons on demand, when they are strongly confined in zerodimensional structures $[9,10]$. To date, most compact sources of single, indistinguishable photons are based on single quantum dots (QDs) integrated in tailored photonic environments [11]. While those systems have been optimized with respect to their efficiencies and photon interference visibilities, a fully scalable production of such devices is still a challenge due to the commonly applied randomized QD growth techniques. In contrast to semiconductor QD-microcavity systems, a polaritonic single photon source would ideally not suffer from very strong inhomogeneous broadening effects and hence could clearly outperform these existing systems in the sense of scalable realization. This would be highly beneficial for linear optics quantum information processing [12]. Indeed, the experimental realization of a system that exhibits polariton blockade (and hence generates polariton number states) would put a large number of goals in the emerging field of solid state based quantum emulation within reach. For instance, reaching the Mott insulator phase of a bosonic system, which is a key requirement to simulate quantum phase transitions [13], strongly relies on the abovementioned effects.

Unfortunately, the prerequisites on the system for the observation of the required polariton quantum blockade effect are rather demanding, and conclusively the demonstration is still elusive.

One important challenge towards the realization of polariton single photon sources is related to the required tight spatial trapping of polaritons. Following the theoretical analysis by
Verger et al. [9] and $\mathrm{Na}$ and Yamamoto [10], a trapping potential of several millielectron volts in the zero detuning range, along with a spatial extension of less than $1 \mu \mathrm{m}$, is required to approach the blockade regime. Trapping of polaritons can be achieved via several techniques, either by trapping the exciton or the photon part. Unfortunately, most gentle (or nondestructive) techniques only allow for the realization of trapping potentials either in the submillielectron volt range (i.e., the evaporation of metal masks on top of a two-dimensional Fabry-Pérot cavity [14]) or do not allow for trapping carriers down to the submicron range (i.e., the mechanical strain technique to trap excitons [3], surface acoustic waves [15]). Large trapping potentials can be obtained, e.g., by etching micropillars through the entire polariton Bragg structure [16,17], and even tighter confinements can be realized by trapping polaritons in photonic crystal cavities [18]. Despite polariton lasing having been observed with both approaches, the rather large surface recombination velocities in etched III/V based QWs [19] and etching induced QW broadenings limit the applicability for the observation of true quantum effects. A much more promising technique to deeply confine polaritons in a small spatial trap is based on a local elongation of a quasiplanar microcavity, such as first demonstrated by El Daiff et al. [20].

In these locally elongated structures, the exciton polaritons are trapped via their photonic component. However, in contrast to regular micropillar cavities, the photonic traps are shallowly etched into the cavity spacer by some nanometers, without affecting or damaging the QW itself. After the etching step, the top distributed Bragg reflector (DBR) is grown to accomplish the Fabry-Pérot cavity. Therefore, detrimental surface recombination effects of QW excitons and etching induced QW broadening are of minor influence in such structures, even in the submicron trap diameter range. The trapping potential provided by this technique can easily exceed several meV, depending on the etch depth. 
A second difficulty related to the observation of polariton blockade is the requirement of strictly resonant pumping the polaritons with the excitation laser. Such techniques usually suffer from laser stray light, interfering with the sample signal when normal incidence conditions are chosen. Especially on the single-photon level, detection of the sample signal in the background of laser stray light is a big challenge. Here, we propose photocurrent (PC) readout techniques to detect small polariton signals from such complex microcavity structures subject to strict resonant excitation. While PC measurements have been performed on planar polariton samples, we go one step beyond and combine such a scheme to detect polariton signals from the mesa trap. The effect of polariton blockade likely cannot be directly manifested in such a scheme due to the continuous PC generated by the pump beam and its incapability to carry out photon number counting. However, as we show in this paper it is suitable to resonantly probe energy shifts of weak polaritonic resonances, and it is certainly a useful approach to study coherent effects in resonantly driven quantum systems [21].

Third, the exciton-photon detuning is a critical parameter in order to observe polariton blockade, as the polariton interactions scale with the affiliated excitonic content [9]. Fine-tuning for standard two-dimensional polariton samples can be performed via the wafer position dependent cavity energy, where single traps are fabricated along the cavity thickness gradient. Temperature tuning is also possible, but with temperature the system parameters are also changed (especially the influence of phonons), while the tuning range is limited to $\sim 1 \mathrm{meV}$. As an alternative route, we demonstrate that we can utilize Stark tuning of the QW, even in a sophisticated polariton trap geometry, as an effective and precise tuning knob for this critical parameter without dramatically reducing the QW oscillator strength.

In order to manipulate and probe the polaritons in our device, we use a fabrication and contacting scheme introduced elsewhere [22]. The device operation principle is depicted in Fig. 1(a): The quasiplanar microcavity with the polariton mesa trap in its center is contacted by a ring with a diameter of $8 \mu \mathrm{m}$ surrounding the spatially elongated region of the cavity. By applying a bias voltage $V_{B}$ between the ring and the backside contact, we can tune the QW emission into the desired cavity-exciton detuning regime. Furthermore, we can also probe the PC flowing through our $p-i-n$ device, when the structure is excited with an external laser.

The employed PC setup is briefly sketched in Fig. 1(b). We use a tunable narrowband continuous wave (CW) diode laser at normal incidence to inject carriers in our device with a variable energy (monitored via a wavemeter) in the range of the polariton resonances. The PC is read out via the electrical contacts of the sample and detected by a picoampere meter.

At first, we will demonstrate that we can simultaneously tune the QW resonance via the quantum confined Stark effect (QCSE), while using PC spectroscopy [23] as a readout mechanism: Fig. 2(a) shows PC spectra for a device with a strong blue detuning of minimal $\Delta=E_{C}-E_{X} \sim 6.5 \mathrm{meV}$, which results in a high excitonic Hopfield coefficient of $|X|^{2} \sim 0.96$. The uncoupled exciton resonance (labeled as $\mathrm{X}$ in the plot) appears as a peak in the $\mathrm{PC}$ spectrum with a linewidth of $\sim 1.8 \mathrm{meV}$, indicating the persistence of a
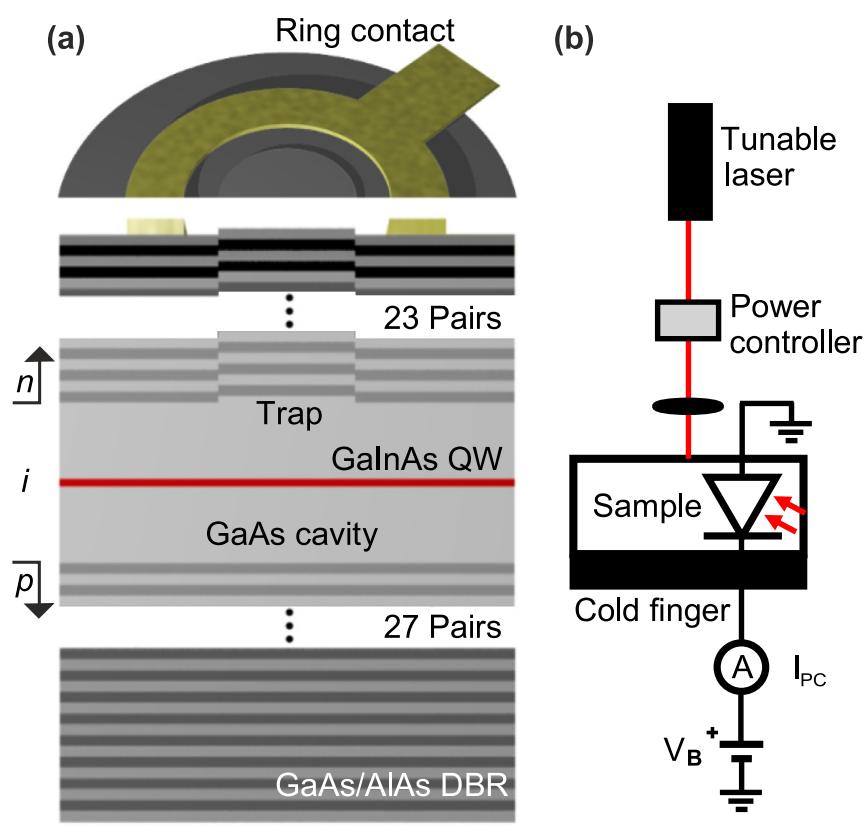

FIG. 1. (Color online) (a) Schematic sketch of the sample design. The type of doping is labeled at the left, the doping concentration is ramped down towards the intrinsic cavity. The QW is drawn in red color. (b) Scheme of the PC setup.

high optical quality. When the applied voltage is increased, a continuous blueshift of the excitonic emission line is caused by the QCSE as the build-in potential $V_{b i}$ is compensated, and the electric field inside the intrinsic $L_{\text {cav }}$-thick cavity layer decreases with $F=\left(V_{b i}+V_{B}\right) / L_{\text {cav }}$. The overall tuning range of this technique is limited in our case to $\sim 10 \mathrm{meV}$; however, this range can be easily extended by optimizing the QW confinement. Consistently, when the flat-band condition is approached and the applied voltage $V_{B}$ approaches $-V_{b i}$ $\left(V_{B}=-0.7 \mathrm{~V}\right.$ to $-1.0 \mathrm{~V}$, depending on the injection power), the absolute PC $I_{\mathrm{PC}}$ exponentially decreases due to the suppressed tunneling rate of electrons out of the QW [inset Fig. 2(a)]. This dependence is given via

$$
I_{\mathrm{PC}}=-e \cdot \alpha_{\tau} \cdot n_{X},
$$

where the population of excitons $n_{X}$ is driven by the applied laser power $P_{\text {laser }}$ via $n_{X}=\eta \cdot T \cdot P_{\text {laser }} / E_{\mathrm{LP}} . E_{\mathrm{LP}}$ herein denotes the energy of the lower polariton. The transmission $T$ and absorption probability $\eta$ both depend on the detuning, which changes through the QCSE. The introduced factor of proportionality $\alpha_{\tau}$ depends on related tunneling times for electron and holes and is approximated assuming steady-state conditions [24]:

$$
\alpha_{\tau}=\frac{2}{\tau_{t, e}}+\frac{1}{\tau_{t, h}}\left(\frac{1}{1+\frac{\tau_{t, e}}{\tau_{N R}}}+1\right) \sim \frac{2}{\tau_{t, e}} .
$$

Hence, it can be expressed (for the given QW parameters $\tau_{t, e} \sim 0.02 \tau_{t, h} \sim 0.001 \tau_{N R}$ ) via the tunneling time $\tau_{t}$ of an electron out of the $\mathrm{QW} \alpha_{\tau}=2 / \tau_{t, e}$ [25]:

$$
\tau_{t,(e, h)}=\frac{2 m_{e, h}^{*} L_{\mathrm{QW}}^{2}}{\hbar \pi} \operatorname{Exp}\left(\frac{4}{3 \hbar e F} \sqrt{2 m_{e, h}^{*} U_{e, h}^{3}}\right) .
$$




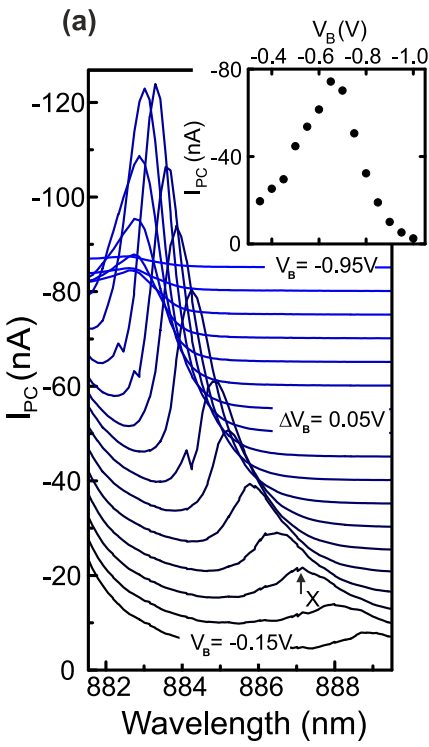

(c)

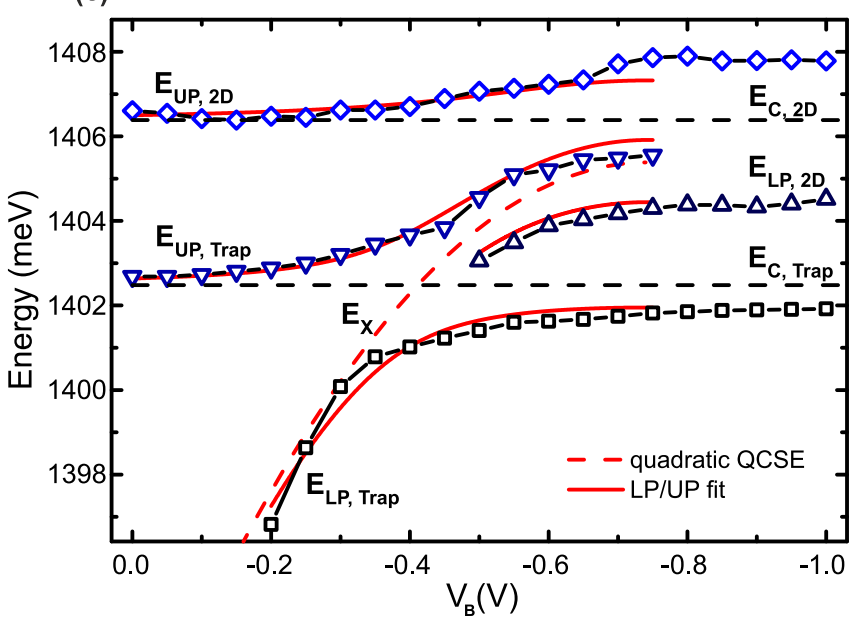

FIG. 2. (Color online) (a) PC spectra of the uncoupled QW with a large positive detuning showing an pronounced quadratic QCSE shift towards flatband (towards higher $V_{B}$ ). Inset of (a) shows the dependence of the extracted peak height on the external bias. (b) Anticrossing behavior of the QW with the photonic resonance of a $2 \mu \mathrm{m}$ trap and the planar background, tuned via the QCSE. Same symbols as in (c) are used to mark the four corresponding energies. (c) PC peak energy extracted from (b), plotted against external bias. Red lines show a global fit based on quadratic QW shift, while dashed lines indicate the uncoupled QW and photon energy.

The effective masses $m_{e, h}^{*}$, the QW width $L_{\mathrm{QW}}$, and the binding energies $U_{e, h}$ are given through the material composition. An increase in PC [inset Fig. 2(a), $V_{B}=-0.3 \mathrm{~V}$ to $-0.7 \mathrm{~V}$ ], on the other hand, can be explained by enhanced absorption because of electron and hole wavefunction overlap and higher sample transmission.

The general behavior changes drastically when the QW approaches the cavity resonance. In Fig. 2(b), this scenario is fulfilled for a $2-\mu \mathrm{m}$-diameter trapping device where the detuning for the trapped ground state can be varied from $+5 \mathrm{meV}\left(|X|^{2} \sim 0.94\right)$ to $-3 \mathrm{meV}\left(|X|^{2} \sim 0.13\right)$. Due to the tight photonic confinement, the $\mathrm{PC}$ splits into a set of zero-dimensional polaritonic resonances, which feature the expected avoided crossing behavior. Due to their flat dispersion, the trapped polariton modes appear as sharp Lorentzian peaks in the PC, while the planar resonator modes from the environment exhibit a short wavelength tail due to their two-dimensional momentum dispersion. Due to the high signal-to-noise ratio and the strict resonant excitation scheme, we can conveniently detect all polariton branches related to the trapped system and the planar system in the background simultaneously at all available detunings. This is a major advantage, e.g., for photo- or electroluminescence spectroscopy, where some of the branches usually are thermally depleted and others are hidden in the luminescence background.

Figure 2(c) depicts the extracted mode energies of our complex system as a function of the applied voltage together with lower polariton (LP) and upper polariton (UP) branches reproduced through a coupled oscillator model considering a quadratic QW shift $[25,26] E_{X}=E_{X, f}+\beta \cdot\left(V_{B}+V_{b i}^{*}\right)^{2}$, where $E_{X, f}$ denotes the flatband energy of $E_{X, f}=1.4054 \mathrm{eV}$ and $\beta=-0.026 \mathrm{eV} \mathrm{V}^{-2}$. The resonance case between the QW and the mesa trap ground mode is reached at an applied voltage of $V_{B}=-0.41 \mathrm{~V}$, giving rise to the upper and lower zero-dimensional polariton branch with a characteristic Rabi splitting of $2.7 \mathrm{meV}$. Subsequently, at higher $V_{B}$, the QW shifts towards resonance with the two-dimensional photon mode.

The parameter used for the effective build-in potential $V_{b i}=$ $0.7 \mathrm{~V}$ at this given laser power is smaller than the theoretically expected build-in potential $V_{b i} \sim 1.3 \mathrm{~V}$. The deviation can be caused by several effects such as screening of intracavity and intra-QW electric field [27,28] or density dependent effects such as polariton-polariton interaction and bleaching of strong coupling. Consequently, we repeated this bias-series analysis for successive lower laser powers and found a closer value of $V_{b i}^{*} \sim 1.19 \mathrm{~V}$ for the undisturbed system by extrapolation of the phenomenological parameters.

In order to study the density dependent behavior of the trapped polaritons around zero detuning, the voltage was set to a value of $V_{B}=-0.4 \mathrm{~V}$. The polaritons are injected with a laser of a constant energy, $\sim 1 \mathrm{meV}$ blue detuned of the LP branch, and the PC of the device is measured. This experiment is repeated at three different detunings (varying by $0.2 \mathrm{meV}$ ) between the injection laser and the LP branch. In all measurement configurations, a pronounced nonlinearity of the PC can be observed, however, appearing at significantly different injection powers, as depicted in Fig. 3. The nonlinearities can be attributed to a polariton density dependent blueshift of the LP branch: While increasing the population in the LP branch, its energy continuously blueshifts until it tunes into resonance with the injection laser. Once this resonance case is reached, the injection efficiency is drastically increased, giving rise to the strong increase of the PC. Consequently, the nonlinearity appears for significantly larger laser powers at larger detunings.

In order to investigate the interactions in our device more quantitatively, we performed energy resolved power dependent PC measurements at different applied voltages. The energy of the trapped LP PC peaks recorded at increasing laser powers are plotted in Fig. 4(a). We observe a pronounced energy shift towards higher energies, which converges towards the uncoupled photonic energy in the high density limit. The signature of the shift is highly dependent on $V_{B}$ as we 


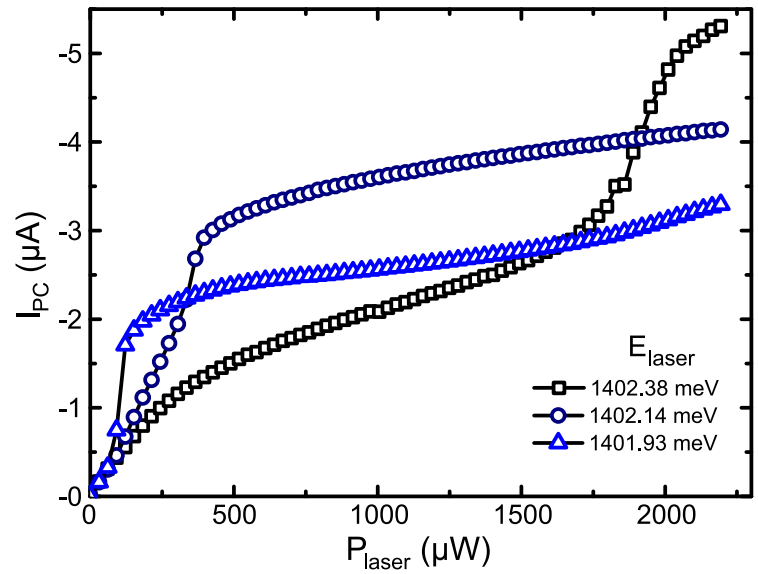

FIG. 3. (Color online) PC versus laser power. The laser injection energy remains constant at three different values slightly blue detuned of the trapped LP for $V_{B}=-0.4 \mathrm{~V}$. Self-tuning into resonance gives rise to the pronounced nonlinearity in the $\mathrm{PC}$ response.

vary the detuning from $\Delta=+3.2 \mathrm{meV}\left(V_{B}=-0.25 \mathrm{~V}\right)$ to $\Delta=+2.5 \mathrm{meV}\left(V_{B}=-0.40 \mathrm{~V}\right)$, which results in a higher polariton density because of higher transmission and absorption probability. Moreover, the loss of carriers is reduced as $\tau_{t, e}$ increases from $V_{B}=-0.25 \mathrm{~V}$ to $V_{B}=-0.40 \mathrm{~V}$.

From the same data, we analyze the energy versus the $I_{\mathrm{PC}}$ output in the low density regime in Fig. 4(b) to get rid of transmission and absorption characteristics. In this regime, the blueshift can be well reproduced by a linear behavior with a slope of $1.3 \times 10^{4} \mathrm{eV} \mathrm{A}^{-1}$ to $8.5 \times 10^{4} \mathrm{eV} \mathrm{A}^{-1}$.

In order to assess the possibility to observe polariton blockade effects in our device, one needs to calculate the energy shift of the LP resonance $\Delta E_{\mathrm{LP}}$ per polariton, normalized to the LP linewidth $\delta E_{\mathrm{LP}}$. Once the energy shift per single polariton exceeds the polariton linewidth (i.e., this factor exceeds unity), single photon operation from the device can be expected. To probe this value in our given device, we further analyzed the blueshift of the $V_{B}=-0.25 \mathrm{~V}$ curve. Figure 4(c) shows the normalized energy shift versus the polariton number, estimated by using Eqs. (1)-(3), assuming that the population of excitons is equal to the population of LPs. The nonradiative recombination time of electrons [24] $\tau_{N R}$ is set to $200 \mathrm{~ns}$. We furthermore set $V_{b i}=(1.19 \pm 0.02) \mathrm{V}$ (extracted from experimental data, see above), $U_{e}=69 \mathrm{meV}$, and $U_{h}=48 \mathrm{meV}$, yielding $\tau_{t, e}=0.21 \mathrm{~ns}$ and $\tau_{t, h}=12 \mathrm{~ns}$. We can extract a value of $\frac{\Delta E_{\mathrm{LP}}}{\Delta N_{P} \cdot \delta E_{\mathrm{LP}}} \sim 1.3 \times 10^{-2}$, i.e., less than two orders of magnitude below the desired threshold. It is worth noting that optimization of the device design (e.g., by vertically aligning the excitons), however, could allow for blueshifts per polaritons on the order of one. Several effects in our device can cause a blueshift of the polariton resonance: the most important are polariton-polariton repulsive interaction, phase-space filling [29] and the QCSE resulting from electric field screening by the tunneling carriers. As the latter is difficult to quantitatively express, we compare the observed blueshift with a calculated LP blueshift theoretically expected by polariton-polariton interaction [30] [black line in Fig. 4(c)]

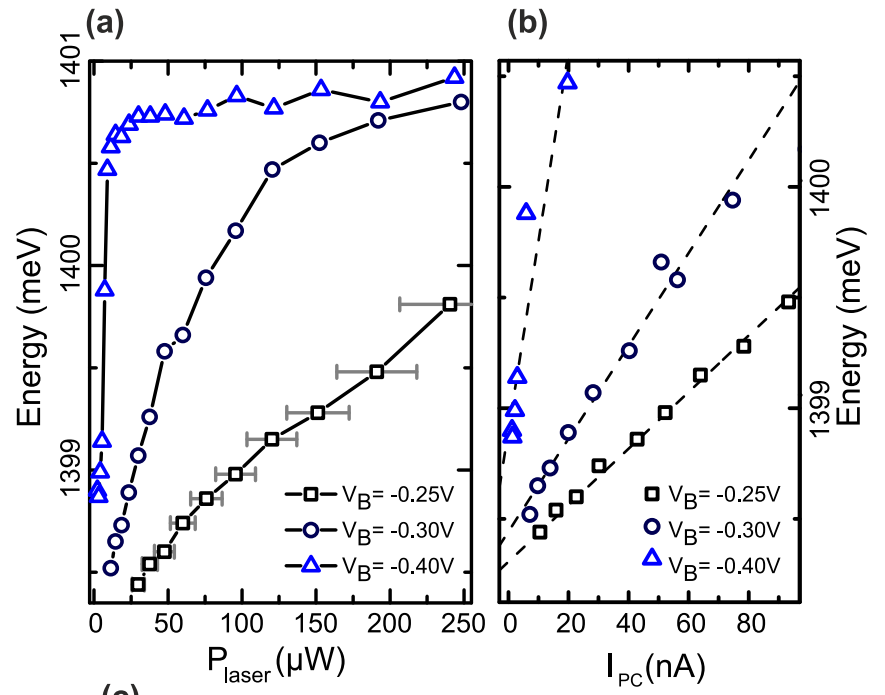

(c)

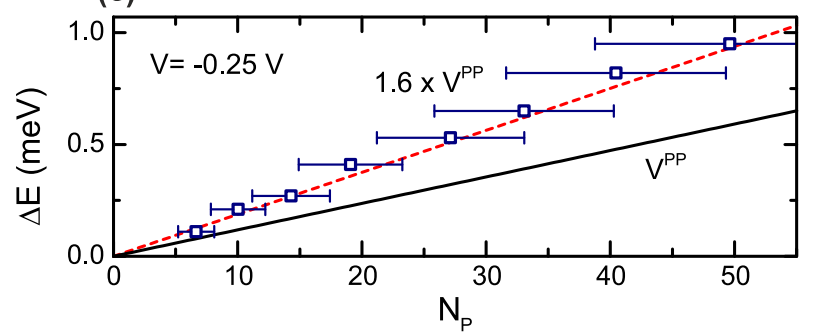

FIG. 4. (Color online) (a) Energy shift of the LP with applied laser power at three different bias and therefore different detunings. The error on applied power for this measurement is indicated for $V_{B}=$ $-0.25 \mathrm{~V}$. (b) Lower polariton energy position in dependence of the photocurrent peak amplitude. (c) Blueshift of the $\operatorname{LP}\left(V_{B}=-0.25 \mathrm{~V}\right)$ with respect to the extrapolated low density value as a function of the calculated polariton population. A linear fit of the blueshift behavior (red dashed line) is exceeding the calculated blueshift attributed to polariton-polariton interaction (black solid line).

using

$$
\Delta E_{\mathrm{LP}}=V^{P P} X_{h}^{4} N_{\mathrm{LP}}=\frac{1}{S} \cdot \frac{3 e^{2} a_{B}}{\epsilon} X_{h}^{4} \cdot N_{\mathrm{LP}} .
$$

We neglect phase-space filling terms as we are operating two orders of magnitude below the polariton population, necessary for reaching the saturation regime with the carrier occupation number $N_{\text {sat }}=S \cdot n_{\text {sat }}=S \cdot \frac{7}{16 \pi a_{B}^{2}} \sim 3 \times 10^{3}$. Here, $S=r^{2} \pi$ is the cross-sectional area of the system, namely the area of the circular trap. Other parameters used are the exciton Bohr radius $a_{B}=12 \mathrm{~nm}$ and $\epsilon=13.9 \epsilon_{0}$ with $\epsilon_{0}$ as the vacuum permittivity. The Hopfield coefficient $X_{h}$ was calculated from known values of Rabi splitting $\left(E_{R}=\right.$ $2.7 \mathrm{meV})$ and photonic mode energy $\left(E_{C}=1.4025 \mathrm{eV}\right)$ by [30]

$$
X_{h}=\left(1+\frac{1}{4}\left(\frac{E_{R}}{E_{\mathrm{LP}}-E_{C}}\right)^{2}\right)^{-\frac{1}{2}} .
$$

The result of the calculation is shown in Fig. 4(c) as a solid black line. It is obvious that the observed blueshift is about $60 \%$ higher than the calculated shift expected solely from polaritonpolariton-interaction. That leads to the conclusion that a 
tunneling carrier induced feedback enhances the blueshift in our system.

In conclusion, we have demonstrated PC measurements on a low-dimensional polariton trap under strictly resonant excitation conditions. This versatile technique is proven highly suitable to study such complex polaritonic devices in the low density and high density limits, while the system can be simultaneously manipulated and fine-tuned via the applied bias. In the low density regime, we have determined an energy shift per polariton of $\sim 1.3 \times 10^{-2} \delta E_{\mathrm{LP}}$ in our device with a diameter of $2 \mu \mathrm{m}$ and identified polariton-polariton repulsive interaction and field screening as the dominating mechanisms.
We anticipate that this value can be straightforwardly increased by the following means: (a) decrease of the polariton trap size down to the submicron range, as proposed by Verger et al. [9]; (b) implementation of exciton polaritons with an increased repulsive interaction. Polaritons with a permanent dipole moment are anticipated to comprise two orders of magnitude higher repulsive interaction strength [31], which would already be sufficient for the observation of polariton blockade effects in our device.

This paper has been supported by the Free State of Bavaria. The authors thank Y. Yamamoto for fruitful discussions.
[1] H. Deng, G. Weihs, C. Santori, J. Bloch, and Y. Yamamoto, Science 298, 199 (2002).

[2] J. Kasprzak, M. Richard, S. Kundermann, A. Baas, P. Jeambrun, J. M. J. Keeling, F. M. Marchetti, M. H. Szymanska, R. André, J. L. Staehli, V. Savona, P. B. Littlewood, B. Deveaud-Plédran, and L. S. Dang, Nature 443, 409 (2006).

[3] R. Balili, V. Hartwell, D. Snoke, L. Pfeiffer, and K. West, Science 316, 1007 (2007).

[4] S. Christopoulos, G. Baldassarri Höger von Högersthal, A. Grundy, P. G. Lagoudakis, A. V. Kavokin, J. J. Baumberg, G. Christmann, R. Butté, E. Feltin, J. F. Carlin, and N. Grandjean, Phys. Rev. Lett. 98, 126405 (2007).

[5] T.-C. Lu, Y.-Y. Lai, Y.-P. Lan, S.-W. Huang, J.-R. Chen, Y.-C. Wu, W.-F. Hsieh, and H. Deng, Opt. Express 20, 5530 (2012).

[6] S. Kéna-Cohen and S. R. Forrest, Nat. Photonics 4, 371 (2010).

[7] K. S. Daskalakis, S. A. Maier, R. Murray, and S. Kéna-Cohen, Nat. Mater. 13, 271 (2014).

[8] J. D. Plumhof, T. Stöferle, L. Mai, U. Scherf, and R. F. Mahrt, Nat. Mater. 13, 247 (2014).

[9] A. Verger, C. Ciuti, and I. Carusotto, Phys. Rev. B 73, 193306 (2006).

[10] N. Na and Y. Yamamoto, New J. Phys. 12, 123001 (2010).

[11] C. Santori, M. Pelton, G. Solomon, Y. Dale, and Y. Yamamoto, Phys. Rev. Lett. 86, 1502 (2001).

[12] P. Kok, K. Nemoto, T. C. Ralph, J. P. Dowling, and G. J. Milburn, Rev. Mod. Phys. 79, 135 (2007).

[13] I. Bloch, J. Dalibard, and W. Zwerger, Rev. Mod. Phys. 80, 885 (2008).

[14] N. Y. Kim, K. Kusudo, C. Wu, N. Masumoto, A. Löffler, S. Höfling, N. Kumada, L. Worschech, A. Forchel, and Y. Yamamoto, Nat. Phys. 7, 681 (2011).

[15] E. A. Cerda-Méndez, D. Sarkar, D. N. Krizhanovskii, S. S. Gavrilov, K. Biermann, M. S. Skolnick, and P. V. Santos, Phys. Rev. Lett. 111, 146401 (2013).

[16] T. Gutbrod, M. Bayer, A. Forchel, J. P. Reithmaier, T. L. Reinecke, S. Rudin, and P. A. Knipp, Phys. Rev. B 57, 9950 (1998).
[17] D. Bajoni, P. Senellart, E. Wertz, I. Sagnes, A. Miard, A. Lemaître, and J. Bloch, Phys. Rev. Lett. 100, 047401 (2008).

[18] S. Azzini, D. Gerace, M. Galli, I. Sagnes, R. Braive, A. Lemaître, J. Bloch, and D. Bajoni, Appl. Phys. Lett. 99, 111106 (2011).

[19] J. Dreybrodt, A. Forchel, and J. P. Reithmaier, Phys. Rev. B 48, 14741 (1993).

[20] O. El Daï, A. Baas, T. Guillet, J.-P. Brantut, R. I. Kaitouni, J. L. Staehli, F. Morier-Genoud, and B. Deveaud-Plédran, Appl. Phys. Lett. 88, 061105 (2006).

[21] A. Zrenner, E. Beham, S. Stufler, F. Findeis, M. Bichler, and G. Abstreiter, Nature, 418, 612 (2002).

[22] K. Winkler, C. Schneider, J. Fischer, A. Rahimi-Iman, M. Amthor, A. Forchel, S. Reitzenstein, S. Höfling, and M. Kamp, Appl. Phys. Lett. 102, 041101 (2013).

[23] D. Bajoni, E. Semenova, A. Lemaître, S. Bouchoule, E. Wertz, P. Senellart, S. Barbay, R. Kuszelewicz, and J. Bloch, Phys. Rev. Lett. 101, 266402 (2008).

[24] D. Bajoni, E. Semenova, A. Lemaître, S. Barbay, R Kuszelewicz, and J. Bloch, Appl. Phys. Lett. 97, 091107 (2010).

[25] G. Bastard, J. A. Brum, and R. Ferreira, Solid State Phys. 44, 229 (1991)

[26] D. A. B. Miller, D. S. Chemla, T. C. Damen, A. C. Gossard, W. Wiegmann, T. H. Wood, and C. A. Burrus, Phys. Rev. Lett. 53, 2173 (1984).

[27] T. Kuroda and A. Tackeuchi, J. Appl. Phys. 92, 3071 (2002).

[28] T. Fujita, T. Toizumi, Y. Nakazato, A. Tackeuchi, T. Chinone, J. H. Liang, and M. Kajikawa, Phys. Status Solidi 5, 356 (2007).

[29] L. Ferrier, E. Wertz, R. Johne, D. D. Solnyshkov, P. Senellart, I. Sagnes, A. Lemaître, G. Malpuech, and J. Bloch, Phys. Rev. Lett. 106, 126401 (2011).

[30] C. Ciuti, P. Schwendimann, and A. Quattropani, Semicond. Sci. Technol. 18, S279 (2003).

[31] P. Cristofolini, G. Christmann, S. I. Tsintzos, G. Deligeorgis, G. Konstantinidis, Z. Hatzopoulos, P. G. Savvidis, and J. J. Baumberg, Science 336, 704 (2012). 